Rev. Élev. Méd. vét. Pays trop., 1978, 31 (2) : 213-218.

\title{
Fermentation microbienne de produits végétaux destinés à l'alimentation du bétail au Sénégal
}

\author{
1. Etude bactériologique et biochimique
}

\author{
par J. BLANCOU (*) \\ (avec la collaboration technique de A. NDOYE et A. NIANG)
}

\begin{abstract}
RÉSUMÉ
L'étude de la fermentation microbienne in vitro des cinq principaux produits végétaux utilisables pour l'alimentation du bétail sénégalais a été réalisée par l'analyse de ses produits finals (bactéries et leurs métabolites). La détermination du taux des acides gras volatils produits s'est avérée la meilleure technique d'étude, puisqu'elle est en corrélation hautement significative avec la valeur U. F. du produit fermenté : ceci peut permettre un test rapide de valeur nutritive des aliments destinés aux ruminants. Cette étude a démontré également l'importance de la charge microbienne initiale de l'aliment dont l'augmentation (par pré-fermentation de l'aliment) pourratt améliorer sa digestibilité.
\end{abstract}

\section{INTRODUCTION}

La fermentation microbienne des produits d'origine végétale est un des phénomènes biologiques les plus anciennement connus, et exploités à des fins diverses, par l'homme.

Certaines de ces fermentations, lorsqu'elles surviennent in vivo dans l'appareil digestif des herbivores, conduisent à des réactions métaboliques également bien connues, et qui constituent la base physiologique de leur nutrition.

Mais, curieusement, si l'un et l'autre de ces aspects de la fermentation végétale ont été chacun bien explorés, leurs inter-relations n'ont pas encore fait l'objet d'une recherche spéciale.

L'attention a été attirée récemment sur ce sujet par MAC CULLOUGHS et collab. (4) qui écrivent, en conclusion d'une étude sur les ensilages $« .$. The data demonstrate a series of interactions between fermentation in the silo, rumen

(*) Laboratoire National de l'Elevage et de Recherches Vétérinaires. B. P. 2057, Dakar. fermentation, silage digestibility and animal performances $»$.

Le but de notre travail a donc été d'étudier, sur le plan bactériologique et biochimique, l'évolution de la fermentation in vitro des principaux aliments d'origine végétale consommés par les ruminants (au Sénégal) et tenter d'en déduire les conséquences sur leur valeur nutritive in vivo.

La seconde partie de l'étude sera consacrée aux conséquences de la pré-fermentation in vitro des aliments sur leur digestibilité et sur les performances des ruminants qui les consomment.

\section{MATERIEL ET MÉTHODES}

\section{Produits végétaux utilisés}

Les 5 principaux produits d'origine végétale utilisés, ou susceptibles d'être utilisés, pour alimenter le bétail sénégalais sont :

- le pâturage naturel graminéen (de composi- 
tion et de qualités variables selon les régions et les saisons) ;

- la fane d'arachide (partie végétative du plant, subsistant après la récolte des graines);

- la paille de riz ou de mil (résidu du battage des épis) ;

- la coque d'arachide (résidu du décorticage industriel des graines).

\section{Méthodes de fermentation}

La fermentation est assurée soit par la population bactérienne d'origine naturellement fixée sur le produit, soit par addition d'une microflore supplémentaire d'origine ruminale (provenant d'un même ruminant donneur, un zébu «Gobra» nourri à la fane d'arachide et muni d'une fistule permanente).

La fermentation ne se produit que lors de la réhydratation du produit étudié par de l'eau contenant 6,5 p. 1000 de chlorure de sodium, selon les proportions suivantes:

produit à fermenter (fragmente) : $20 \mathrm{~g}$ bruts $(*)$ eau salée 6,5 p. $1000( \pm 10$ p. 100 d'inoculum bactérien) : q. s. p. : $200 \mathrm{~g}(* *)$.

Ce mélange, bien homogénéisé, est placé dans un ballon stérile de $500 \mathrm{ml}$, fermé hermétiquement (avec évacuation possible des gaz formés) puis étuvé $48 \mathrm{~h}$ à $37^{\circ}$. La fermentation qui se produit est donc aéro-anaérobie.

\section{Méthode d'analyse des produits de fermentation}

Après les $48 \mathrm{~b}$ d'incubation, toute la fraction liquide contenue dans le ballon est récupérée par essorage et filtration sur gaze puis soumise aussitôt aux analyses suivantes:

- Biochimiques: ces techniques, classiques, ont été décrites antérieurement (2) :

- mesure du $\mathrm{pH}$ au $\mathrm{pH}$ mètre électrique;

- dosage de l'ammoniac par la méthode de

(*) Le taux de matières sèches moyen du produit est déterminé sur le même échantillon pour permettre de rapporter ultérieurement les résultats au poids sec.

(**) Cet inoculum, constitué par la phase liquide du contenu ruminal, contient en moyenne $10^{8}$ à $10^{\circ}$ bactéries aéro-anaérobies vivantes par gramme. Il est indispensable si le produit à fermenter a été stérilisé (par chauffage, traitement chimique, etc...).
Conway (les résultats sont indiqués en milligrammes par litre);

- dosage des acides acétiques, propioniques et butyriques par chromatographie en phase gazeuse (les résultats sont indiqués en grammes par litre).

- Bactériologiques : Numération des bactéries aéro-anaérobies strictes par dilution logarithmique de la suspension et culture en gélose nutritive additionnée de 5 p. 100 d'extrait de rumen stérile (3) sur :

- 2 boîtes de Pétri incubées à $37^{\circ}$ en aérobiose ;

- 2 boîtes de Pétri incubées à $37^{\circ}$ en atmosphère de $\mathrm{CO}_{2}$ et $\mathrm{H}_{2}$ (jarre type «Gas-Pak»).

Les colonies sont dénombrées après $72 \mathrm{~h}$, à la loupe, sur les boîtes ensemencées avec les dilutions permettant un dénombrement correct.

\section{RÉSULTATS}

Les résultats sont figurés sous forme d'un tableau général (1) indiquant, pour chaque produit, les valeurs des différentes analyses selon que la fermentation a eu lieu spontanément (en présence de la seule microflore attachée au substrat), ou après addition d'une microflore exogène. Le taux de matières sèches du produit est également inđiqué, ce qui permet de rapporter les résultats de l'analyse au poids sec.

En ce qui concerne le pâturage naturel, dont la composition évolue au cours de l'année $\left({ }^{*}\right)$, les analyses ont été faites mensuellement. Toutes les valeurs indiquées sont les moyennes de plusieurs observations ( $8 \mathrm{au}$ minimum) et suivies de l'indication de leur intervalle de confiance au seuil 0.05 p. 100 (tableau II).

Dans les 2 tableaux, l'analyse statistique indique une différence significative ou hautement significative entre les valeurs trouvées pour les différents produits (tableau I) ou les différentes périodes (tableau II).

(*) Remarque : C'est pourquoi toutes nos analyses ont été effectuées sur des échantillons prélevés sur la même parcelle de pâturage naturel (dunes de la région des Niayes, dans la presqu'île du Cap-Vert). Sur cette parcelle dominaient les graminées des genres Andropogon, Cenchrus, Digitaria, Dactyloctenium. 


\begin{tabular}{|c|c|c|c|c|c|c|c|c|}
\hline 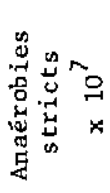 & $\begin{array}{l}m \\
n \\
+1 \\
\infty \\
-1\end{array}$ & $\begin{array}{l}n \\
5 \\
+1 \\
\approx\end{array}$ & $\begin{array}{l}m \\
\dddot{y} \\
+1 \\
-1\end{array}$ & 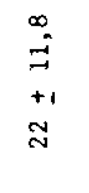 & $\begin{array}{l}m \\
n \\
+1 \\
0\end{array}$ & $\begin{array}{l}0 \\
5 \\
+1 \\
0\end{array}$ & $\begin{array}{l}N \\
n \\
+1 \\
n\end{array}$ & $\begin{array}{l}-5 \\
+1 \\
+1 \\
\infty\end{array}$ \\
\hline 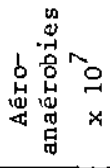 & $\begin{array}{l}m \\
\infty \\
+1 \\
N\end{array}$ & $\begin{array}{l}m \\
m \\
+1 \\
\Rightarrow\end{array}$ & $\begin{array}{l}m \\
\text { on } \\
+1 \\
\infty \\
0\end{array}$ & 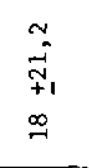 & $\begin{array}{l}a \\
n \\
+1 \\
r \\
n\end{array}$ & $\begin{array}{l}\infty \\
j \\
+1 \\
a\end{array}$ & $\begin{array}{l}\vec{a} \\
+1 \\
a\end{array}$ & $\begin{array}{l}m \\
m \\
0\end{array}$ \\
\hline $\begin{array}{l}m \\
x^{m}\end{array}$ & $\begin{array}{l}\stackrel{0}{\vec{n}} \vec{\eta} \\
\vec{r} \\
+1\end{array}$ & $\begin{array}{cc}0 & m \\
0 & 0 \\
+1 & 0 \\
+1\end{array}$ & 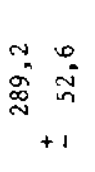 & 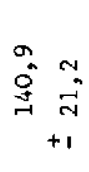 & $\begin{array}{cc}\infty & \infty \\
\sigma^{n} & 0 \\
& +1\end{array}$ & 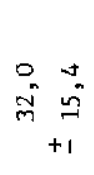 & $\begin{array}{cc}\infty & \infty \\
0 & \infty \\
\infty & \infty \\
& +1\end{array}$ & $\begin{array}{cc}\text { v } & 0 \\
0 & 0 \\
\text { in } & \infty \\
+1\end{array}$ \\
\hline 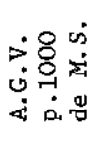 & {$\left[\begin{array}{l}\vec{\sigma} \\
-i\end{array}\right]$} & \begin{tabular}{|l|}
$g$ \\
0 \\
$m$ \\
\end{tabular} & {$\left[\begin{array}{l}5 \\
\sigma \\
-1\end{array}\right.$} & {$\left[\begin{array}{l}0 \\
0 \\
0 \\
0\end{array}\right.$} & {$\left[\begin{array}{c}0 \\
0 \\
0 \\
0\end{array}\right]$} & $\begin{array}{c}9 \\
1 \\
0 \\
0\end{array}$ & \begin{tabular}{|l|}
$m$ \\
0 \\
0 \\
\end{tabular} & \begin{tabular}{|l|} 
\\
\\
0
\end{tabular} \\
\hline 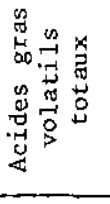 & $\begin{array}{c}\stackrel{n}{\stackrel{0}{r}} \stackrel{0}{-} \\
-1 \\
0 \\
+1\end{array}$ & $\begin{array}{c}n \\
N \\
N \\
n \\
+1\end{array}$ & $\begin{array}{cc}0 & 0 \\
\infty & m \\
-1 & 0 \\
+1\end{array}$ & 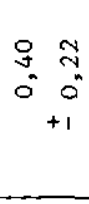 & \begin{tabular}{cc}
8 & $\infty$ \\
\multirow{2}{*}{} & 0 \\
0 & 0 \\
0 & 0 \\
+1
\end{tabular} & $\begin{array}{cc}N & \stackrel{2}{N} \\
0 & 0 \\
& 0 \\
+1\end{array}$ & 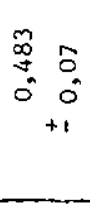 & $\begin{array}{cc}9 & n \\
0 & 0 \\
0 & 0 \\
& 0\end{array}$ \\
\hline 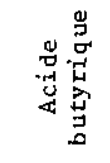 & 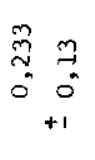 & 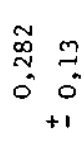 & $\begin{array}{cc}\stackrel{n}{\infty} & \overrightarrow{0} \\
\stackrel{1}{0} & 0 \\
0 & 0 \\
& +1\end{array}$ & 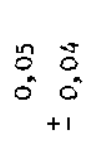 & 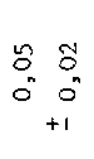 & $\begin{array}{c}20 \\
\overrightarrow{0} \\
0 \\
0 \\
+1\end{array}$ & $\begin{array}{c}\stackrel{0}{\infty} \\
\stackrel{0}{+} \\
0 \\
0 \\
+1\end{array}$ & $\begin{array}{cc}\stackrel{\text { Ln }}{\circ} & -1 \\
0 & 0 \\
0 & 0\end{array}$ \\
\hline 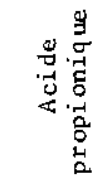 & 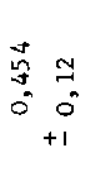 & $\begin{array}{l}\stackrel{\circ}{\circ} \\
\therefore 0 \\
+1\end{array}$ & \begin{tabular}{c}
$N$ \\
\multirow{0}{0}{} \\
0 \\
+1
\end{tabular} & \begin{tabular}{cc}
2 & $g$ \\
\hdashline & 0 \\
0 & 0 \\
+1
\end{tabular} & 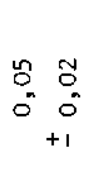 & $\begin{array}{cc}0 & \infty \\
0 \\
0 & 0 \\
& 0 \\
+1\end{array}$ & 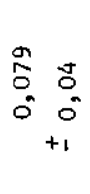 & $\begin{array}{cc}n & n \\
0 & 0 \\
0 & 0 \\
+1\end{array}$ \\
\hline 岂 & $\begin{array}{cc}0 & \pm \\
-1 & 0 \\
+1\end{array}$ & $\begin{array}{c}\infty \\
m \\
-1 \\
-1\end{array}$ & 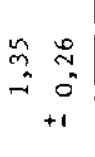 & \begin{tabular}{cc}
$\infty$ & $\infty$ \\
\hdashline & 0 \\
0 & 0 \\
0 & 0 \\
& +1
\end{tabular} & $\begin{array}{cc}\stackrel{9}{0} & 0 \\
0 \\
0 & 0 \\
0 & 0 \\
& +1\end{array}$ & 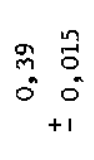 & \begin{tabular}{cc}
\multirow{J}{J}{} & 0 \\
0 & 0 \\
0 & 0 \\
+1
\end{tabular} & $\begin{array}{cc}\infty & m \\
m & n \\
0 & 0 \\
& +1\end{array}$ \\
\hline 票 & \begin{tabular}{c}
$\circ$ \\
$\stackrel{9}{9}$ \\
\multirow{0}{0}{0} \\
+1
\end{tabular} & $\begin{array}{cc}\hat{0} & 0 \\
+ & 0 \\
+1\end{array}$ & $\begin{array}{c}\approx \\
\approx \\
i \\
+1\end{array}$ & \begin{tabular}{cc}
0 & $\infty$ \\
\hdashline & 0 \\
+1
\end{tabular} & 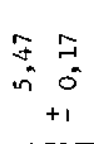 & $\begin{array}{cc}0 & 0 \\
\text { in } & 0 \\
+1\end{array}$ & $\begin{array}{c}\text { in } 0 \\
\text { in } \\
\text { t } \\
+1\end{array}$ & $\begin{array}{c}\text { N } \\
\text { m } \\
\text { ñ } \\
+1\end{array}$ \\
\hline 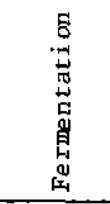 & $\omega$ & 4 & co & $\varangle$ & תם & $\varangle$ & $n$ & $<$ \\
\hline 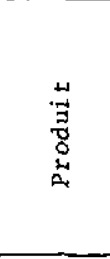 & 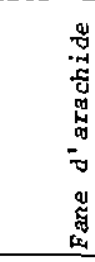 & $\begin{array}{l}\text { Oे } \\
\stackrel{2}{0} \\
\infty \\
11 \\
11 \\
\dot{\Sigma} \\
\dot{\Sigma}\end{array}$ & 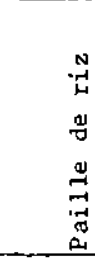 & $\begin{array}{l}\hat{m} \\
\tilde{a} \\
\tilde{n} \\
11 \\
\dot{j} \\
\dot{\Sigma}\end{array}$ & 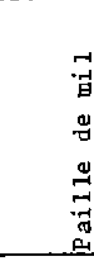 & 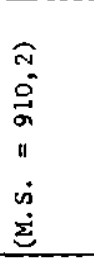 & 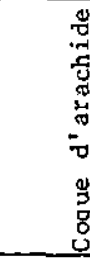 & $\begin{array}{l}\text { 子 } \\
\text { m } \\
\text { a } \\
n \\
\dot{2} \\
\dot{\Xi}\end{array}$ \\
\hline
\end{tabular}


TABL. $\mathrm{N}^{\circ}$ II-Analyse de la fermentation du pâturage naturel selon les saisons.

\begin{tabular}{|c|c|c|c|c|c|c|c|c|c|}
\hline Mois & $\mathrm{pH}^{\mathrm{H}}$ & $\begin{array}{c}\text { Acide } \\
\text { acétique }\end{array}$ & $\begin{array}{c}\text { Acide } \\
\text { propionique }\end{array}$ & $\begin{array}{c}\text { Acide } \\
\text { butyrique }\end{array}$ & $\begin{array}{c}\text { Acides gras } \\
\text { volatils } \\
\text { totaux }\end{array}$ & $\begin{array}{l}\text { A.G.V. } \\
\text { P. } 1000 \text { de } \\
\text { M.S. }\end{array}$ & $\mathrm{N} \mathrm{H}_{3}$ & $\begin{array}{l}\text { Aēro-anaérobies } \\
\text { par m1 x } 10^{7}\end{array}$ & $\begin{array}{l}\text { Anaérobies } \\
\text { stricts par } \\
\text { m1 } \times 10^{7}\end{array}$ \\
\hline $\begin{array}{l}\text { Janvier } \\
(M S=904)\end{array}$ & $5,28 \pm 0,20$ & $0,55 \pm 0,22$ & $0,105 \pm 0,08$ & $0,08 \pm 0,08$ & $0,72 \pm 0,26$ & 0,80 & $73,6 \pm 52,3$ & $0,645 \pm 0,413$ & $0,577 \pm 0,43$ \\
\hline $\begin{array}{l}\text { Février } \\
(M S=898)\end{array}$ & $5,40 \pm 0,31$ & $0,50+0,11$ & $0,104 \pm 0,027$ & $0,09 \pm 0,0$ & $0,69 \pm 0,15$ & 0,77 & $86,4 \pm 39,8$ & $0,65 \pm 0,28$ & $0,72 \pm 0,5$ \\
\hline $\begin{array}{l}\text { Mars } \\
(M S=913)\end{array}$ & $5,50 \pm 0,22$ & $0,43 \pm 0,05$ & $0,09 \pm 0,05$ & $0,101 \pm 0,0$ & $0,62 \pm 0,09$ & 0,68 & $111,4 \pm 13,5$ & $0,60 \pm 0,1$ & $0,52 \pm 0,2$ \\
\hline $\begin{array}{l}\text { Avri1 } \\
(\mathrm{MS}=914)\end{array}$ & $5,58 \pm 0,81$ & $0,43 \pm 0,16$ & $0,09 \pm 0,05$ & $0,08 \pm 0,01$ & $0,60 \pm 0,10$ & 0,66 & $121,3 \pm 29,7$ & $0,32 \pm 0,1$ & $0,27 \pm 0,1$ \\
\hline $\begin{array}{l}\text { Mai } \\
(\mathrm{MS}=917)\end{array}$ & $5,71 \pm 0,96$ & $0,41 \pm 0,3$ & $0,09 \pm 0,05$ & $0,08 \pm 0,08$ & $0,58 \pm 0,38$ & 0,63 & $93,9 \pm 97$ & $0,29 \pm 0,14$ & $0,23 \pm 0,09$ \\
\hline $\begin{array}{l}\text { Juin } \\
(\text { MS }=917)\end{array}$ & $5,48 \pm 1,9$ & $0,43 \pm 0,1$ & $0,08 \pm 0,07$ & $0,08 \pm 0,01$ & $0,61 \pm 0,20$ & 0,67 & $112,9 \div 114,6$ & $0,6 \pm 0,3$ & $\pm 0,29$ \\
\hline $\begin{array}{l}\text { Juillet } \\
(\mathrm{MS}=165,1)\end{array}$ & $5,82 \pm 2,2$ & $0,52 \pm 0,19$ & $0,08 \pm 0,04$ & $0,06 \pm 0,25$ & $0,68 \pm 1,50$ & 4,12 & $178,6 \pm 101,6$ & $\pm 1,9$ & $2,75 \pm 0,95$ \\
\hline $\begin{array}{l}\text { Août } \\
(M S=202,8)\end{array}$ & $5,20 \pm 1,09$ & $0,58 \pm 0,19$ & $0,135 \pm 0,13$ & $0,20 \pm 0,17$ & $0,95 \pm 0,19$ & 4,68 & $106,7 \pm 60,6$ & $2,7 \pm 2,8$ & $1,35 \pm 1,2$ \\
\hline $\begin{array}{l}\text { Sep temb re } \\
(\mathrm{MS}=340,4)\end{array}$ & $4,86 \pm 0,42$ & $0,38 \pm 0,14$ & $0,092 \pm 0,12$ & $0,28 \pm 0,05$ & $0,69 \pm 0,32$ & 2,03 & $87,95 \pm 20,6$ & $\pm 2,9$ & $\pm 2,5$ \\
\hline $\begin{array}{l}\text { Octob re } \\
(M S=505,3)\end{array}$ & $5 \pm 0,43$ & $0,26 \pm 0,1$ & $0,10 \pm 0,07$ & $0,16 \pm 0,04$ & $0,57 \pm 0,14$ & 1,13 & $36,45 \pm 20,92$ & $2,1 \pm 0,8$ & $\pm 2,1$ \\
\hline $\begin{array}{l}\text { Novembre } \\
(\mathrm{MS}=840,1)\end{array}$ & $5,17 \pm 0,33$ & $0,75 \pm 0,23$ & $0,41 \pm 0,10$ & $0,011 \pm 0,04$ & $1,05 \pm 0,45$ & 1,25 & $122,7 \pm 65,7$ & $\pm 1,7$ & $\pm 1,3$ \\
\hline $\begin{array}{l}\text { Dé cembre } \\
(\mathrm{M} \varphi \text { }=882,6)\end{array}$ & $5,26 \pm 0,14$ & $0,54 \pm 0,27$ & $0,10 \pm 0,06$ & $0,07 \pm 0,02$ & $0,70 \pm 0,37$ & 0,79 & $95,65 \pm \quad 28,8$ & $\pm 2,1$ & $\pm 0,9$ \\
\hline
\end{tabular}




\section{CONCLUSION - DISCUSSION}

1. Choix du paramètre le mieux adapté à l'étude de la fermentation

Les critères de ce choix doivent raisonnablement être, pour chacun des paramètres étudiés :

- un écart net des résultats entre 2 produits différents :

- une fluctuation (intervalle de confiance) faible entre les résultats concernant un même produit.

L'examen des 2 tableaux conduit alors à chojsir le dosage des acides gras volatils plutôt que la variation $\mathrm{du} \mathrm{pH}$, le taux d'ammoniac ou le nombre des bactéries anaérobies

Le dosage considéré sera de préférence celui qui a été obtenu après fermentation spontanée (ou mieux, fermentation du produst préalablement stérilisé, puis additionné de l'inoculum). On peut, en effet, constater que l'addition des 10 p. 100 d`inoculum à un produit non stérile conduit parfois à des résultats aberrants, ce quı avast déjà été constaté par H. A. BLADEN (1). Le résultat de ce dosage sera évidemment ramené au taux de matières sèches du produit.

\section{Relation entre dosages des acides gras volatils et valeur nutritive des aliments}

Il était logıque d'espérer constater une relation entre la valeur nutritive d'un aliment pour le ruminant et la quantıté d'acides gras volatils que peut dégager la fermentation in vitro du même aliment, puisqu'il s'agit d'un processus microbien assez semblable.

L'examen du tableau III confirme cette attente. puisqu'on constate une relation évidente entre la valeur (en U.F.) moyenne et le taux des acides gras volatils produits.
TABLEAU $\mathrm{N}^{\bullet}$ III

\begin{tabular}{|l|c|c|}
\hline \multicolumn{1}{|c|}{ Produit étudié } & $\begin{array}{r}\text { Taux d'acides } \\
\text { gras volatils }\end{array}$ & Valeur U.F. $*$ \\
\hline Fane d'arachide & 1,80 à 2,00 & 0,30 à 0,40 \\
\hline Coque d'arachide & 0,46 à 0,60 & 0 \\
\hline Paille de riz 0,08 \\
\hline Paille de mil & 1,63 à 2,35 & 0,35 à 0,45 \\
\hline $\begin{array}{l}\text { Pãturage de } \\
\text { saison sèche }\end{array}$ & 0,36 à 0,52 & 0,05 à 0,15 \\
\hline $\begin{array}{l}\text { Pâturage de saison } \\
\text { des pluies }\end{array}$ & 0,47 à 1,70 & 0,10 à 0,30 \\
\hline
\end{tabular}

Valeur du coefficient de corrélation : $r=0,94$ (significatif à P.0.,01)

:Les deux valeurs indiquées sont les valeurs extrêmes déterminées au cours de différentes analyses réalisées sur des échantı1lons de même provenance.

\section{Applications pratiques}

Deux applications pratiques découlent des précédentes observations :

- Il pourrait devenir possible de déterminer rapidement la valeur U.F. comparée de différents aliments du bétail par simple titrage des acides gras volatils libérés par sa fermentation (n) vitro. Ceci nécessiterait l'établissement des tables de détermination étudiées dans des cond1tions précises de fermentation.

- La charge bactérienne initıale jouant un rôle prépondérant dans la production d'acides gras volatils il doit être possible d'augmenter cetfe production par addition d'une microflore supplémentaire (au cours de la pré-fermentation de l'aliment) pour améliorer la digestibilité in vivo : ce sera l'objet de la seconde partie de notre étude.

\section{SUMMAR Y}

Microbial fermentation of vegetal products for cattle reeding in Senegal Bacteriological and biochemical study

Study of rumen fermentation m viro, on five vegetal products usually fed to senegalese cattle, was achteved by evaluation of microbial development and volatile fatty acid production. This last method seems better, highly correlated with U. F. amount, and could be used for rapid determination of nutritional value.

The data demonstrate importance of fecd-attached mucrobial population, which could be developped (in pre-fermentation) to improve digestibility of vegetal products. 


\section{RESLMEN}

\section{Fermentación microbiana de productos vegetales para la alimentación del ganado en Senegal. 1 Estudio bacteriologico y bioquimico}

Se realizó el estudio de la fermentación microbiana in vitro de los cinco pruncipales productos vegetales utilızables para la alimentación del ganado senegalés por el analisis de sus productos finales (bacterias y sus metabolitos). El mejor técnico de estudio fué la determinación de la tasa de los ácidos grasos volátiles producidos, pues que es en correlación con el valor $\mathrm{U}$. F. del producto fermentado : lo que puede permitir una prueba rápida de valor nutritivo de los alimentos para los rumiantes. Este estudio demostró tambien que el aumento del número inicial de microbıos en el alimento (por pre-fermentación del alimento) podria mejorar su digestıbilıdad.

\section{BIBLIOGRAPHIF}

1. BLADEN (H. A.), DOETSCH (R. N.). Physiological activities of rumen mixed all suspensions. J. Agric. Appl. Chem., 1959 (7) : 791-794.

2. CALVET (H.), BOUDERGUES (R.). REMESY (C.), ARCHAMBAULT DE VENCAY (J.). Recherches sur le métabolisme du rumen chez les bovins tropicaux. Rev. Elev. Méd. vét. Pays trop., 1971, 24 (2) : 287-296.
3. HUNGATE (R. E.). The rumen and its microbes, New York, Academic Press, 1966.

4. Mac CULLOUGHS (M. E.), SISK (L. R.), SMART (W. W. G.). Influence of fermentation in the silo on rumen fermentation, silage intake and digestibility. $J$. dairy Sci., 1970, 53 (8) : 1042-1045. 